\title{
Diffusion of spheres in crowded suspensions of rods
}

\author{
Kyongok Kang \\ Forschungszentrum Jülich, Institute für Festkörper Forschung (IFF), Weiche Materie, \\ D-52425 Jülich, Germany \\ J. Gapinski \\ Institute of Physics, A. Mickiewicz University, Umultowska 85, 61-614 Poznan, Poland
}

M. P. Lettinga, J. Buitenhuis, and G. Meier

Forschungszentrum Jülich, Institute für Festkörper Forschung (IFF), Weiche Materie, D-52425 Jülich, Germany

M. Ratajczyk

Institute of Physics, A. Mickiewicz University, Umultowska 85, 61-614 Poznan, Poland

Jan K. G. Dhont

Forschungszentrum Jülich, Institute für Festkörper Forschung (IFF), Weiche Materie, D-52425 Jülich, Germany

A. Patkowski

Institute of Physics, A. Mickiewicz University, Umultowska 85, 61-614 Poznan, Poland

(Received 27 July 2004; accepted 26 October 2004; published online 10 January 2005)

Translational tracer diffusion of spherical macromolecules in crowded suspensions of rodlike colloids is investigated. Experiments are done using several kinds of spherical tracers in fd-virus suspensions. A wide range of size ratios $L / 2 a$ of the length $L$ of the rods and the diameter $2 a$ of the tracer sphere is covered by combining several experimental methods: fluorescence correlation spectroscopy for small tracer spheres, dynamic light scattering for intermediate sized spheres, and video microscopy for large spheres. Fluorescence correlation spectroscopy is shown to measure long-time diffusion only for relatively small tracer spheres. Scaling of diffusion coefficients with $a / \xi$, predicted for static networks, is not found for our dynamical network of rods (with $\xi$ the mesh size of the network). Self-diffusion of tracer spheres in the dynamical network of freely suspended rods is thus fundamentally different as compared to cross-linked networks. A theory is developed for the rod-concentration dependence of the translational diffusion coefficient at low rod concentrations for freely suspended rods. The proposed theory is based on a variational solution of the appropriate Smoluchowski equation without hydrodynamic interactions. The theory can, in principle, be further developed to describe diffusion through dynamical networks at higher rod concentrations with the inclusion of hydrodynamic interactions. Quantitative agreement with the experiments is found for large tracer spheres, and qualitative agreement for smaller spheres. This is probably due to the increasing importance of hydrodynamic interactions as compared to direct interactions as the size of the tracer sphere decreases. (C) 2005 American Institute of Physics. [DOI: 10.1063/1.1834895]

\section{INTRODUCTION}

There is a large body of literature on diffusion of spheres and, to a lesser extent, of rods. Much less is known about diffusion of spheres in host dispersions of rodlike particles, which is both of biological and technological importance.

Due to the relevance of long-time self-diffusion for mass transport in cells, the majority of diffusion experiments of this kind reported so far are on self-diffusion of proteins in suspensions of $F$ actin, both in vitro and in vivo. ${ }^{1-10}$ Contrary to $F$-actin networks in vivo, where many actin-binding proteins regulate actin polymerization, $F$-actin networks in vitro are (sometimes cross linked) networks of relatively long filaments with a highly dispersed length. Uncontrolled bundling of filaments is sometimes found and continuous polymerization and depolymerization of the filaments occurs (for an overview of properties of actin and the role played by bind- ing proteins, see Ref. 11). The dynamics of $F$-actin networks is so slow, that it does not couple to the diffusion of tracer spheres. This leads to scaling of the diffusive properties with $a / \xi$ (where $a$ is the tracer sphere radius and $\xi$ the mesh size of the $F$-actin network). As will be seen, such a scaling is not found for highly dynamical networks of rods, such as the fd-virus suspensions investigated in the present paper.

Anomalous diffusion in $F$-actin networks and the cell interior has been found in Refs. 4, 7, 8, and 10, where a tracer sphere resides for some time within a "cage" formed by the network, and occasionally "jumps" from one cage to the other. Due to the very slow dynamics of the cross linked or strongly entangled $F$-actin networks, a spherical Brownian particle is captured in a quasistatic cage from which it escapes on a time scale that is much larger than the time the tracer needs to diffuse over distances comparable to the size of each cage (typical length and time scales for various dy- 
namical processes in actin solution can be found in Ref. 12). $F$-actin networks are thus quite complicated by themselves. To gain in fundamental understanding of transport of spherical Brownian particles through highly dynamical networks, where the dynamics of the network couples to the diffusive properties of a tracer sphere, other types of host systems are therefore more appropriate.

In four very recent papers, diffusion of spheres in host suspensions of slender particles, other than $F$ actin, have been reported: nucleosome core particles in dispersions of DNA, ${ }^{13}$ colloidal spheres in solutions of "living polymers", ${ }^{14}$ colloidal spheres in dispersions of xanthan, ${ }^{15}$ and polymer spheres in dilute suspensions of tobacco mosaic virus. ${ }^{16}$ Diffusion of the nucleosome core particles in DNA is in part controlled by "histone tails" that fold out of the protein core on increasing ionic strength. These histone tails interact specifically with DNA, and lead to adsorption of the nucleosome core particle on DNA. Adsorption leads to anomalous, "stretched exponential," diffusive behavior and an almost discontinuous jump of the diffusion coefficient at the ionic strength where unfolding occurs. ${ }^{13}$ These experiments were done using fluorescence correlation spectroscopy, which for the small protein probably probes long-time diffusion. Short- and long-time translational diffusion of tracer spheres (with radii 125 and $250 \mathrm{~nm}$ ) in a host dispersion living polymers have been discussed in Ref. 14. The living polymers consist of self-associating monomers bis(ethylhexylureido)toluene (EHUT). Both the polymerization/ depolymerization kinetics of EHUT and the length distribution of the living polymers probably have a strong influence on the diffusive properties of the tracer spheres. Short-time translational and rotational diffusion of a tracer sphere with a radius of $93 \mathrm{~nm}$ in high molecular weight xanthan dispersions has been studied in Ref. 15. The xanthan used in this study is a relatively flexible molecule (contour length $2 \mu \mathrm{m}$ and persistence length $120 \mathrm{~nm}$ ). Diffusion in these xanthan solutions is probably comparable to diffusion in polymer networks. In Ref. 16, diffusion of polymer spheres and tobacco mosaic virus (TMV) particles in their mixtures with low TMV concentration have been studied. Here, the emphasis is on the salt concentration dependence of diffusion coefficients of both species. The dependence of diffusion coefficients on the concentration of both species has not been probed in these experiments.

In the present study we use fd virus as a rodlike colloidal host particle. These are relative stiff rods, which are chemically inert without any specific interactions with the tracer spheres. Fd-virus suspensions have been recognized before as very good model system for rod dispersions in connection to (micro) rheology ${ }^{17-20}$ and phase transitions (sometimes of binary mixtures with spherical colloids or polymers). ${ }^{21-24}$

There are a number of theories on translational diffusion of tracer spheres through networks. ${ }^{25-28}$ In all these theories it is assumed that the network can be described as being fixed in space. This results in the above mentioned $a / \xi$ scaling, which is shown to be invalid for dynamical networks of rods (see Ref. 14 and the present paper). So far, there is no theory that incorporates the dynamics of the network itself on diffusion of tracer spheres. An exception are a mode- coupling theory ${ }^{29}$ and simulations ${ }^{30}$ on diffusion close to the isotropic-nematic phase transition, where slowing down of orientational diffusion is a necessary condition for the validity of the mode-coupling approach.

In the present paper we report on experiments on tracer diffusion in dynamical networks of rigid, chemically inert fd-virus rods, and propose a theory for low concentrations of rods, where Brownian motion of the rods is accounted for. The theory is discussed in Sec. II. Its validity is limited to concentrations below the overlap concentration, but might be extended (in future work) to describe diffusion in highly entangled dynamical networks as well. The colloidal systems are introduced in Sec. III. Fd-virus particles are used as host particles (contour length $880 \mathrm{~nm}$, thickness $6 \mathrm{~nm}$, and persistence length $2.2 \mu \mathrm{m}$ ), while the radii of the colloidal spheres vary between 3 and $500 \mathrm{~nm}$. Due to this large range of tracer sizes we needed various techniques to probe tracer diffusion. These experimental techniques are discussed in some detail in Sec. IV. Experimental results are presented in Sec. V, and are compared to the theoretical predictions for low rod concentrations.

\section{THEORY: A VARIATIONAL APPROACH FOR LOW ROD CONCENTRATIONS AND BIG SPHERES}

In the theory discussed here, the assumption will be that the tracer sphere interacts with uncorrelated rods at each instant of time, that is, with rods which do not mutually interact with each other. This limits the validity of the theory to rod concentrations below the overlap concentration. Hydrodynamic interactions between the tracer sphere and the rods are neglected in the present treatment. In a future extension of the theory, hydrodynamic interactions and correlations between rods might be included.

\section{A. The variational approach}

Little is known about long-time self-diffusion of spheres in host dispersions of rigid, Brownian rods. A variational approach that has been successfully applied to self-diffusion of rods ${ }^{31}$ will be formulated here for self-diffusion of spheres in suspensions of rods, to leading order in concentration of the rods. This approach leads to predictions for the coefficient $\alpha$ in an expansion of the long-time self-diffusion coefficient $D_{s}$ of the spheres with respect to the volume fraction $\varphi$ of the rods,

$$
D_{s}=D_{0}\left[1-\alpha \varphi+O\left(\varphi^{2}\right)\right]
$$

where $D_{0}=k_{B} T / 6 \pi \eta a$ is the Einstein diffusion coefficient of a single, noninteracting sphere with radius $a$ in solvent with shear viscosity $\eta$. The variational approach presented here renders numerical values for $\alpha$ as a function of the rod aspect ratio ( $L$ is the length and $D$ the thickness of the rods),

$$
p=L / D,
$$

and the ratio of the length of the rod and the diameter $2 a$ of the sphere,

$$
q=L / 2 a \text {. }
$$




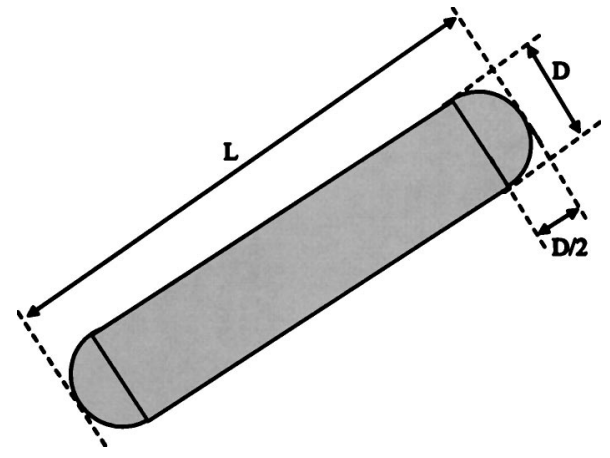

FIG. 1. An end-capped rod: a cylinder capped on both ends by a semisphere with a diameter equal to the thickness of the cylinder. $L$ is the total length of the rod and $D$ its thickness.

Here we shall neglect hydrodynamic interactions between the tracer sphere and the rods. This can be done when the sphere and rod are not "massive" bodies compared to each other. That is, the sphere should not be larger than the rod length (that is $2 a \leqslant L$ ), while the rod diameter should not be larger than the sphere diameter (that is $D \leqslant 2 a$ ). Hydrodynamic interactions are thus probably not so important in the range,

$$
1 \leqslant q \leqslant p .
$$

Results presented here are limited to this range of size ratios. However, as will be seen later when comparing to experiments, hydrodynamic interactions probably become more important when the size of the tracer sphere is decreased. For a quantitative prediction of the initial rod-concentration dependence of the tracer diffusion coefficient for relatively small tracer spheres, hydrodynamic interactions should be taken into account. The present theory will turn out to agree quantitatively with experiments for relatively large tracer spheres, but only qualitatively for small tracer spheres.

End-capped rods will be considered, where $L$ is the total length of the rod. $L / D=1$ thus corresponds to a sphere with diameter $D$ (see Fig. 1).

The basic idea of the variational approach is as follows. As noted by Batchelor, ${ }^{32}$ the long-time self-diffusion coefficient can be written as $D_{s}=k_{B} T / \gamma$, where the friction coefficient $\gamma$ includes both interactions of the sphere with solvent molecules and the rods. Hence, a weak constant force $\mathbf{F}^{\text {ext }}$ is exerted on the sphere, until the sphere attains a stationary ensemble averaged velocity $\langle\mathbf{v}\rangle$, from which the friction coefficient follows as,

$$
\mathbf{F}^{\mathrm{ext}}=\gamma\langle\mathbf{v}\rangle \text {. }
$$

The calculation of the long-time self-diffusion coefficients thus amounts to calculate the stationary velocity of the sphere due to a constant, weak external force. On the Brownian time scale, and with the neglect of hydrodynamic interactions, this velocity is equal to,

$$
\langle\mathbf{v}\rangle=\beta D_{0}\left[\mathbf{F}^{\text {ext }}-N\left(\left\langle\nabla_{1} V\right\rangle+k_{B} T\left\langle\nabla_{1} \ln \{P\}\right\rangle\right)\right],
$$

to leading order in the concentration of host rods. Here, $\beta$ $=1 / k_{B} T$ (with $k_{B}$ Boltzmann's constant and $T$ the temperature), $N$ is the number of rods in the system under consideration, $V$ the pair-interaction energy of the tracer sphere and the host rods, and $P$ the probability density function (pdf) of the phase space coordinates of the tracer sphere and a rod. Furthermore, $\nabla_{1}$ is the gradient operator with respect to the position coordinate $\mathbf{r}_{1}$ of the tracer sphere. The second term between the rectangular brackets in Eq. (6) is the force that rods exert on the sphere while the third term is the Brownian force on the sphere. The ensemble averaged velocity can be calculated once the pdf $P$ is known. In order to calculate $\alpha$ in Eq. (1), it is sufficient to consider the tracer sphere to interact with a single rod, in which case the pdf $P$ is a function of the position coordinate $\mathbf{r}_{1}$ of the sphere, the position coordinate $\mathbf{r}_{2}$ of the rod, and the orientation $\hat{\mathbf{u}}$ of the rod, which is the unit vector along its long axis. Introducing the dimensionless position coordinates $\mathbf{R}_{j}=\mathbf{r}_{j} / D$ and the corresponding dimensionless gradient operators, $P$ is the solution of the Smoluchowski equation (the external force is taken along the $z$ direction),

$$
0=\left\{\hat{\mathcal{L}}^{(0)}+\beta D F^{\mathrm{ext}} \hat{\mathcal{L}}^{(1)}\right\} P\left(\mathbf{r}_{1}-\mathbf{r}_{2}, \hat{\mathbf{u}}\right),
$$

where the operator $\hat{\mathcal{L}}^{(0)}$ is the Smoluchowski operator without the external force acting on the tracer sphere ( $v$ is an arbitrary phase function),

$$
\begin{aligned}
\hat{\mathcal{L}}^{(0)} v & =\epsilon \nabla_{1} \cdot\left[\nabla_{1} v+\beta v \nabla_{1} V\right]+\nabla_{2} \cdot\left[\nabla_{2} v+\beta v \nabla_{2} V\right] \\
& +\epsilon_{t} \nabla_{2} \cdot\left[\hat{\mathbf{u}} \hat{\mathbf{u}}-\frac{1}{3} \hat{\mathbf{I}}\right] \cdot\left[\nabla_{2} v+\beta v \nabla_{2} V\right] \\
& +\epsilon_{r} \hat{\mathcal{R}} \cdot[\hat{\mathcal{R}} v+\beta v \hat{\mathcal{R}} V],
\end{aligned}
$$

while $\hat{\mathcal{L}}^{(1)}$ accounts for the external force acting on the tracer sphere,

$$
\hat{\mathcal{L}}^{(1)} v=-\epsilon \frac{\partial}{\partial Z} v
$$

where $Z$ is the $z$ coordinate of $\mathbf{R}$. The operator $\hat{\mathcal{R}}=\hat{\mathbf{u}} \times \nabla_{\hat{\mathbf{u}}}$ is the rotation operator, with $\boldsymbol{\nabla}_{\hat{\mathbf{u}}}$ the gradient operator with respect to $\hat{\mathbf{u}}$. The dimensionless numbers $\epsilon, \epsilon_{t}$, and $\epsilon_{r}$ are related to the average Einstein translational diffusion coefficients $D_{0}$ of the sphere and $\bar{D}=\left(2 D_{\perp}+D_{\|}\right) / 3$ of a rod, with $D_{\perp}$ and $D_{\|}$the diffusion coefficients for motion perpendicular and parallel to the rod's long axis, respectively, the difference $\Delta D=D_{\|}-D_{\perp}$, and the Einstein rotational diffusion coefficient $D_{r}$, as,

$$
\begin{aligned}
& \epsilon \equiv D_{0} / \bar{D}=q \frac{1}{\ln \{p\}+0.316+0.582 / p+0.102 / p^{2}}, \\
& \epsilon_{t} \equiv \Delta D / \bar{D}=\frac{3}{4} \frac{\ln \{p\}-1.253+1.775 / p-0.552 / p^{2}}{\ln \{p\}+0.316+0.582 / p+0.102 / p^{2}}, \\
& \epsilon_{r} \equiv D^{2} D_{r} / \bar{D}=\frac{9}{p^{2}} \frac{\ln \{p\}-0.662+0.917 / p+0.078 / p^{2}}{\ln \{p\}+0.316+0.582 / p+0.102 / p^{2}} .
\end{aligned}
$$

These expressions have been shown in Ref. 32 to accurately describe diffusion of single rods for a large range of size ratios.

For small forces, one can write, 


$$
\begin{aligned}
P\left(\mathbf{R}_{1}-\mathbf{R}_{2}, \hat{\mathbf{u}}\right)= & P^{\mathrm{eq}}\left(\left|\mathbf{R}_{1}-\mathbf{R}_{2}\right|, \hat{\mathbf{u}}\right) \\
& \times\left\{1+\beta D F^{\mathrm{ext}} \Psi\left(\mathbf{R}_{1}-\mathbf{R}_{2}, \hat{\mathbf{u}}\right)\right\},
\end{aligned}
$$

where the factor $\beta D$ renders $\Psi$ dimensionless, and $P^{\text {eq }}$ $\sim \exp \{-\beta V\}$ is the equilibrium solution of the Smoluchowski equation without the external force. Substitution of this form into the Smoluchowski equation (7)-(10) yields to leading order in $F^{\text {ext }}$,

$$
\hat{\mathcal{L}}^{(0)}\left[P^{\mathrm{eq}} \Psi\right]=-\hat{\mathcal{L}}^{(1)} P^{\mathrm{eq}} .
$$

This Smoluchowski equation for the perturbation $\Psi$ of the equilibrium pdf due to the external force acting on the tracer sphere is too complicated to solve analytically. Instead we shall discuss a method to obtain a variational solution. The following theorem ${ }^{33}$ will be employed to arrive at a variational solution of the Smoluchowski equation. Consider the functional,

$$
F[v] \equiv\langle v \mid \hat{\mathcal{A}} v\rangle-2\langle v \mid f\rangle,
$$

with $\hat{\mathcal{A}}$ a linear, Hermitian, and negative definite operator with respect to the inner product $\langle\cdots \mid \cdots\rangle$, and with $f$ a known function. The unique function $\Psi$ that maximizes this functional is easily shown to be the solution of the equation $\hat{\mathcal{A}} \Psi=f$. The Smoluchowski equation (13) can be recast in this form as follows. First define the backward Smoluchowski operator $\hat{\mathcal{L}}_{B}^{(0)}$ as the Hermitian conjugate of $\hat{\mathcal{L}}^{(0)}$ with respect to the unweighted inner product $\langle u \| v\rangle$ $\equiv \int d \mathbf{R}_{1} \int d \mathbf{R}_{2} \oint d \hat{\mathbf{u}} u v$, where $\oint$ is the integral over the unit spherical surface, that is, over all orientations $\hat{\mathbf{u}}$ of a rod. By partial integration it can be shown that,

$$
\begin{aligned}
\hat{\mathcal{L}}_{B}^{(0)} v= & \boldsymbol{\epsilon}\left(\nabla_{1}-\beta\left[\nabla_{1} V\right]\right) \cdot \nabla_{1} v+\left(\nabla_{2}-\beta\left[\nabla_{2} V\right]\right) \cdot \nabla_{2} v \\
& +\epsilon_{t}\left(\nabla_{2}-\beta\left[\nabla_{2} V\right]\right) \cdot\left[\hat{\mathbf{u}} \hat{\mathbf{u}}-\frac{1}{3} \hat{\mathbf{I}}\right] \cdot \nabla_{2} v \\
& +\boldsymbol{\epsilon}_{r}(\hat{\mathcal{R}}-\beta[\hat{\mathcal{R}} V]) \cdot \hat{\mathcal{R}} v .
\end{aligned}
$$

The square brackets around $\nabla_{j} V$ and $\hat{\mathcal{R}} V$ are used to indicate that the differentiations are limited to the pair-interaction potential $V$. By substitution it is found that, $\hat{\mathcal{L}}^{(0)}\left[P^{\mathrm{eq}} v\right]$ $=P^{\mathrm{eq}} \hat{\mathcal{L}}_{B}^{(0)} v$. The Smoluchowski equation (13) can thus be rewritten as,

$$
\hat{\mathcal{L}}_{B}^{(0)} \Psi=-\left[P^{\mathrm{eq}}\right]^{-1} \hat{\mathcal{L}}^{(1)} P^{\mathrm{eq}} .
$$

By partial integration it is found that $\hat{\mathcal{L}}_{B}^{(0)}$ is Hermitian with respect to the weighted inner product $\langle u \mid v\rangle$ $\equiv \int d \mathbf{R}_{1} \int d \mathbf{R}_{2} \oint d \hat{\mathbf{u}} P^{\mathrm{eq}} u v$, that is, $\left\langle u \mid \hat{\mathcal{L}}_{B}^{(0)} v\right\rangle=\left\langle\hat{\mathcal{L}}_{B}^{(0)} u \mid v\right\rangle$. Furthermore, the backward Smoluchowski operator is negative definite with respect to the weighted inner product. According to the above mentioned theorem, it follows that the unique function $\Psi$ that maximizes the functional,

$$
F[\Psi] \equiv\left\langle\Psi \mid \hat{\mathcal{L}}_{B}^{(0)} \Psi\right\rangle+2\left\langle\Psi \mid\left[P^{\mathrm{eq}}\right]^{-1} \hat{\mathcal{L}}^{(1)} P^{\mathrm{eq}}\right\rangle,
$$

is the solution of the Smoluchowski equation (13). Substitution of the explicit forms of the operators into Eq. (17) leads to (for mathematical details see Appendix A),

$$
\begin{aligned}
F[\Psi]= & -\int d \mathbf{R} \oint d \hat{\mathbf{u}} \chi\left\{\left(1+\epsilon-\frac{1}{3} \epsilon_{t}\right)|\nabla \Psi|^{2}\right. \\
& \left.+\epsilon_{t}|\hat{\mathbf{u}} \cdot \nabla \Psi|^{2}+\epsilon_{r}|\hat{\mathcal{R}} \Psi|^{2}\right\} \\
& +2 \epsilon \oint d \hat{\mathbf{u}}\left[\int_{R<(L+2 a) / 2 D} d \mathbf{R} \chi \frac{\partial}{\partial Z} \Psi\right. \\
& \left.-\left(\frac{L+2 a}{2 D}\right)^{2} \oint_{R=(L+2 a) / 2 D} d \hat{\mathbf{R}} \frac{Z}{R} \Psi\right]
\end{aligned}
$$

where $\chi$ is $P^{\mathrm{eq}}$, normalized such that $\chi=1$ when the cores do not overlap, while $\chi=0$ when the cores overlap.

\section{B. The trial function}

As a last step, a reasonable trial function $\Psi$ has to be chosen with respect to which the functional (18) should be maximized. For spherical host particles, instead of rods, the exact solution of the Smoluchowski equation at low concentrations reads $\Psi=(\partial / \partial Z) 1 / R$ (see Refs. 34 and 35$)$. This function reflects a decrease of local concentration in the wake of the tracer sphere, and an increase ahead of the sphere. The corresponding value for $\alpha$ in this case is 2 (including hydrodynamic interactions changes this number to 2.10). Note that normalization of the pdf in Eq. (12) requires that,

$$
\int d \mathbf{R} \oint d \hat{\mathbf{u}} \Psi=0
$$

This condition is satisfied by the above mentioned exact solution for a sphere, since it is antisymmetric on reflection in the $x y$ plane. Regarding the rod as a string of spherical beads, a reasonable trial function for rodlike host particles is a sum of the above mentioned solution for a sphere over the beads, that is,

$$
\Psi(\mathbf{R}, \hat{\mathbf{u}})=\frac{\partial}{\partial Z}\left\{a_{1} \frac{1}{R}+a_{2} \int_{-1 / 2[(L / D)-1]}^{1 / 2[(L / D)-1]} d l \frac{1}{|\mathbf{R}-l \hat{\mathbf{u}}|}\right\},
$$

where the sum over beads is replaced by a contour integral. For $L / D=1$, that is for spherical host particles, the integral vanishes and the exact result for spheres is recovered from the first term in the curly brackets. For finite sized rods, both coefficients $a_{1}$ and $a_{2}$ are parameters with respect to which the functional (18) should be maximized. A similar trial function for tracer diffusion of rods has been shown to be reasonably accurate. ${ }^{31}$ In Ref. 31 , two trial functions have been used: a function similar to Eq. (20), and a more advanced trial function, where the connectedness of the beads within the rods is taken into account. The coefficient $a_{2}$ is thereby replaced by an angular and contour-length dependent function, which is taken equal to the first terms in a Taylor expansion with respect to these angular variables and the contour length. For the present purpose, this advanced trial function reads (with $\hat{u}_{z}$ the $z$ component of $\hat{\mathbf{u}}$ ), 


$$
\begin{aligned}
\Psi^{\mathrm{adv}}(\mathbf{R}, \hat{\mathbf{u}})= & \frac{\partial}{\partial Z}\left\{a_{1} \frac{1}{R}+\left[a_{2}+a_{3} Z^{2}+a_{4} \hat{u}_{z}^{2}\right.\right. \\
& \left.+a_{5}(\hat{\mathbf{u}} \cdot \mathbf{R})^{2}\right] \int_{-1 / 2[(L / D)-1]}^{1 / 2[(L / D)-1]} d l \frac{1}{|\mathbf{R}-l \hat{\mathbf{u}}|} \\
& \left.+a_{6} \int_{-1 / 2[(L / D)-1]}^{1 / 2[(L / D)-1]} d l \frac{l^{2}}{|\mathbf{R}-l \hat{\mathbf{u}}|}\right\} .
\end{aligned}
$$

The relative difference between numerical values for $\alpha$ as obtained from the simple trial function (20) and the advanced trial function (21) is a measure for the accuracy of $\alpha$.

The trial function in Eq. (20) is a linear combination of the two functions $\Phi_{1}=\partial(1 / R) / \partial Z$ and $\Phi_{2}$ equal to the derivative of the integral in Eq. (20). Substitution of such a linear combination into Eq. (17) leads to the following expressions for the values of the coefficients that maximize the functional:

$$
a_{i}=-\sum_{j} M_{i j}^{-1} f_{j}
$$

where $M_{i j}^{-1}$ is the inverse of the matrix $M_{i, j}=\left\langle\Phi_{i} \mid \hat{\mathcal{L}}_{B}^{(0)} \Phi_{j}\right\rangle$ and $f_{j}=\left\langle\Phi_{j} \mid\left[P^{\mathrm{eq}}\right]^{-1} \hat{\mathcal{L}}^{(1)} P^{\mathrm{eq}}\right\rangle$. Explicit forms for these matrix and vector elements can be obtained directly from Eq. (18): these correspond to the bilinear and linear contributions in $\Psi$, respectively.

\section{Numerical results}

Once the coefficients $a_{i}$ are found from Eq. (22) (with $i=1,2$ for the simple trial function and $i=1, \cdots, 6$ for the advanced trial function), $\alpha$ can be calculated from,

$$
\begin{aligned}
\alpha= & \frac{1}{\pi^{2} p[1-(1 / 3 p)]} \oint d \hat{\mathbf{u}}\left[\int_{R<(L+2 a) / 2 D} d \mathbf{R} \chi \frac{\partial}{\partial Z} \Psi\right. \\
& \left.-\left(\frac{L+2 a}{2 D}\right)^{2} \oint_{R=(L+2 a) / 2 D} d \hat{\mathbf{R}} \frac{Z}{R} \Psi\right] .
\end{aligned}
$$

Mathematical details of the derivation of this expression from Eqs. (5) and (6) can be found in Appendix B.

The relative difference $\sigma=\left(\alpha^{\mathrm{sim}}-\alpha^{\mathrm{adv}}\right) / \alpha^{\text {adv }}$ for numerical results for $\alpha$ as obtained from the simple trial function (20) and the advanced trial function (21) are plotted in Fig. 2 as a function of $q$ for various aspect ratios $p$. The relative difference is of the order of $10 \%$ for $q \leqslant p<50$, and not larger than about $60 \%$ for $q \leqslant p<200$. The accuracy of numerical values for $\alpha$ as obtained from the advanced trial function is therefore of the order of $30 \%$. In view of the orders of magnitude variation of $\alpha$ as a function of $p$ and $q$, such an accuracy is acceptable.

Numerical values for $\alpha$ as a function of $p=L / D$ and $q$ $=L / 2 a$, obtained from the advanced trial function, were found to be equal to within an accuracy of about $5 \%-10 \%$, to the Padé approximant,

$$
\alpha=2 \frac{p^{2}}{q} \frac{1+\frac{9}{100}(p-1)+\frac{15}{100}(q-1)+\frac{1}{30}(q-1)^{2}+\frac{29}{50000}(p-1)(q-1)^{2}-\frac{7}{50000}(q-1)^{3}}{1+\frac{7}{5}(p-1)-\frac{1}{4}(q-1)+\frac{14}{25}(p-1)(q-1)+\frac{57}{500}(q-1)^{2}+\frac{1}{100}(p-1)(q-1)^{2}} .
$$

In Fig. 3, $\alpha$ is plotted as a function of $q$ for various values of $p$ (with $1 \leqslant q \leqslant p$ ). This figure shows the strong variation of $\alpha$ with $p$ and $q$. Note the very large value of $\alpha$ for large values of $p$ and $q \approx 1$. This implies a strong concentration dependence of the long-time self-diffusion coefficient of a tracer sphere with a diameter that is approximately equal to the length of long and thin host rods. Such a strong concentration dependence is not due to network formation of the rods, since the present theory is only valid below the overlap concentration.

\section{THE COLLOIDAL SYSTEMS}

As tracer spheres we used silica particles (with radii 35, 210 , and $500 \mathrm{~nm}$ ) labeled with Rhodamine B and the protein Bovine serum albumin (BSA) (radius is $3.5 \mathrm{~nm}$ ). The solvent was a $20 \mathrm{mM}$ TRIS/HCl buffer with $p \mathrm{H}=8.15$. Most experiments are done with $100 \mathrm{mM}$ added $\mathrm{NaCl}$. The ionic strength with added salt is $110 \mathrm{mM}$, and without added salt $10 \mathrm{mM}$. The smallest silica particles with a radius of $35 \mathrm{~nm}$ were prepared in several steps, leading to a polydispersity that is much less as for a direct Stöber synthesis. First the core of pure silica is synthesized in a microemulsion. ${ }^{36}$ This dispersion was then coated with Rhodamine B 5-isothiocyanate (RBITC), a layer of about $1.5 \mathrm{~nm}$ thickness, and then covered with two more silica layers of about the same thickness. ${ }^{37}$ For the two largest silica particles with radii of 210 and $500 \mathrm{~nm}$, first a Rhodamine-B labeled core was synthesized, which is then coated with a $100-200 \mathrm{~nm}$ layer of nonfluorescent silica according to Ref. 38, which is a modification of the Stöber synthesis. ${ }^{39}$ Typical dye contents

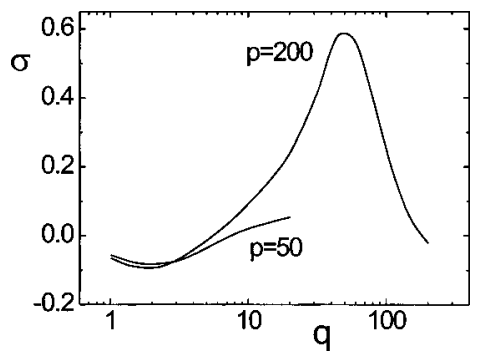

FIG. 2. The relative error $\sigma=\left(\alpha^{\operatorname{sim}}-\alpha^{\text {adv }}\right) / \alpha^{\text {adv }}$ for $\alpha$ as a function of $q$ $=L / 2 a$ for $p=200$ and $p=50$, as indicated in the figure. Here, $\alpha^{\text {sim }}$ and $\alpha^{\text {adv }}$ are the values of $\alpha$ as obtained from the simple trial function (20) and the advanced trial function (21). 
are 1 dye per $(8-13 \mathrm{~nm})^{3}$ for RBITC labeled spheres, corresponding to volume percentages of $0.01 \%-0.1 \%$, and a fluorophore concentration of around $0.3-3 \mathrm{mM}$. For the $35 \mathrm{~nm}$ spheres the dye content was about 1 dye/particle. RBITC has its excitation maximum around $560 \mathrm{~nm}$ and its emission maximum around $580 \mathrm{~nm}$. Typical extinction coefficients are $2.2 \times 10^{4} \mathrm{M}^{-1} \mathrm{~cm}^{-1}$ at $560 \mathrm{~nm}$ (and $1.0 \times 10^{4} \mathrm{M}^{-1} \mathrm{~cm}^{-1}$ at $532 \mathrm{~nm}$ ) for the RBITC labeled particles.

BSA (Serva Feinbiochimica, Germany, product number 11925, Lot 05185) has molecular weight of $66 \mathrm{kDa}$ and a radius of $3 \mathrm{~nm}$. BSA was fluorescently labeled with 5(6) TAMRA-SE [5(6) carboxytetramethylrhodamine, succinimidyl ester] - Sigma-Aldrich, Product number C4759, Lot $100 k 1600$. The dye was connected to the $\mathrm{N}$ terminus of the protein chain using a standard procedure. After the labeling the BSA-TAMRA was purified from the free dye and the BSA aggregates using a Sephadex G-75 column.

The bacteriophage $\mathrm{fd}$ is a rodlike molecule with a contour length of $L=880 \mathrm{~nm}$, a bare diameter of $D=6.6 \mathrm{~nm}$, a persistence length of $P=2200 \mathrm{~nm}$, a molecular weight of $M=1.64 \times 10^{7} \mathrm{~g} / \mathrm{mol}$, and an effective density of $10 \mathrm{e} / \mathrm{nm}$ in water at $p \mathrm{H}=8.15$. $^{40}$ The $\mathrm{fd}$ virus was grown and purified following standard biochemical protocols ${ }^{41}$ using the XL 1 blue strain of E. coli as the host bacteria. The standard yield is ca. $15 \mathrm{mg}$ of fd per liter of infected bacteria, and virus is typically grown in 61 batches. The virus particles were purified by repeated centrifugation $\left(10^{5} \mathrm{~g}\right.$ for $\left.5 \mathrm{~h}\right)$ and finally redispersed in a $20 \mathrm{mM}$ TRIS-HCl buffer at $p \mathrm{H} 8.15$ with $100 \mathrm{mM} \mathrm{NaCl}$ to screen the electrostatic interactions. The buffer contributes about $10 \mathrm{mM}$ to the total ionic strength.

In order to compare experiments with theory, we have to define the thickness of the rods and the radii of the spheres. The Debye length for the TRIS/HCl buffer with added salt is about $0.9 \mathrm{~nm}$. For the larger spheres, where no salt is added, the Debye screening length is $3.0 \mathrm{~nm}$. These screening lengths are so small that DLVO theory is probably not applicable anymore. The effective thickness of charged rods as proposed in Refs. 42 and 43 is therefore questionable. It turns out that a correction of the thickness of the rods does not significantly affect the comparison between experiment and theory: an increase of the effective thickness of the rods decreases $\alpha$ (see Fig. 3) and increases the volume fraction. It turns out that the product $\alpha \varphi$ is relatively insensitive to corrections of the thickness of the rods. We shall therefore simply use the bare thickness of the rods in comparing experiment to theory. In addition, the effective radii of the spheres (except possibly for BSA) is not much different from their bare radii. The aspect ratio of the fd rods is therefore taken equal to,

$$
p=133 \text {. }
$$

Furthermore, from the molar mass $1.64 \times 10^{7} \mathrm{~g} / \mathrm{mol}$, it follows that the volume fraction $\varphi$ of rods can be obtained from the weight concentration $c$ in units $\mathrm{mg} / \mathrm{ml}$ as,

$$
\varphi=\left(1.10 \times 10^{-3}\right)_{c} \times[\mathrm{mg} / \mathrm{ml}] .
$$

These two relations allow the comparison between the theoretical predictions of Sec. III and experiments in Sec. V.

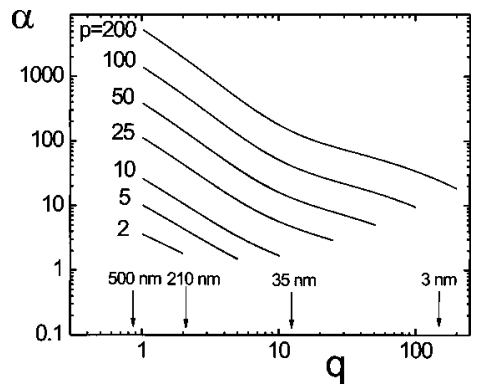

FIG. 3. $\alpha$ as obtained from the advanced trial function as a function of $q$ $=L / 2 a$ for various values of the rod aspect ratio $p=L / D$, as indicated in the figure, for $1 \leqslant q \leqslant p$ [see Eq. (4)]. The fd rods used in the experiments have an aspect ratio of 133 (see Sec. III). The size ratios for the tracer spheres used in our experiments are indicated.

\section{EXPERIMENTAL METHODS}

Depending on the size of the tracer spheres, different methods must be applied to measure the long-time selfdiffusion coefficient. As will turn out, fluorescence correlation spectroscopy (FCS) can only be used for sufficiently small tracer spheres. For larger spheres, the diffusion coefficient as measured with FCS is somewhere in between the long-time and short-time diffusion coefficient. Dynamic light scattering (DLS) can be used when the tracer spheres scatter as least 10-20 times as much as the host rods. This limits the use of DLS to sufficiently large spheres. For very large spheres, however, the scattering angles that should be probed are outside the experimental window where accurate measurements can be done. In addition, the scattered intensity of the host rods dramatically increases at such small scattering angles. For such very large spheres, video microscopy is the more appropriate method to measure self-diffusion coefficients.

In this section we shall discuss FCS, DLS, and video microscopy in some detail.

\section{A. Fluorescence correlation spectroscopy}

In fluorescence correlation spectroscopy a single laser beam is strongly focussed, and the fluorescent intensity from a region around the focal point (the so-called "confocal volume") is probed. The detected intensity distribution within the confocal volume is well described by a Gaussian profile,

$$
I_{0}(\mathbf{r}) \sim \exp \left\{-\left(x^{2}+y^{2}\right) / 2 \sigma_{1}^{2}\right\} \exp \left\{-z^{2} / 2 \sigma_{2}^{2}\right\},
$$

where $\sigma_{1}$ and $\sigma_{2}$ measure the width of the confocal volume in the plane perpendicular to the propagation of the beam and its height along the propagation direction, respectively. Typically, $\sigma_{1} \approx 200 \mathrm{~nm}$, and $\sigma_{2} \approx 800 \mathrm{~nm}$. The fluorescent intensity is equal to,

$$
I_{f}(t) \sim \int d \mathbf{r} I_{0}(\mathbf{r}) c_{d}(\mathbf{r}, t),
$$

where $c_{d}$ is the local concentration of fluorescent dye molecules, which are (at least in part) attached to the colloidal spheres. The time dependence of the fluorescent intensity is entirely due to Brownian motion of spheres in and out of the confocal volume. What is neglected in Eq. (28) is the dependence of excitation probabilities and fluorescent intensities 
on the orientation of dye molecules. This is probably a good approximation when there are many dye molecules with random orientations attached to the colloidal particles. In a FCS experiment, the fluorescent intensity correlation function $C_{f}(t) \equiv\left\langle I_{f}(t) I_{f}(t=0)\right\rangle$ is measured, where the brackets $\langle\cdots\rangle$ refer to ensemble averaging. From Eq. (28) it is found that,

$$
\begin{aligned}
C_{f}(t) & =\int d \mathbf{r} \int d \mathbf{r}^{\prime} I_{0}(\mathbf{r}) I_{0}\left(\mathbf{r}^{\prime}\right)\left\langle c_{d}(\mathbf{r}, t) c_{d}\left(\mathbf{r}^{\prime}, t=0\right)\right\rangle \\
& \sim \int d \mathbf{k} \int d \mathbf{k}^{\prime} I_{0}(\mathbf{k}) I_{0}\left(\mathbf{k}^{\prime}\right)\left\langle c_{d}(\mathbf{k}, t) c_{d}\left(\mathbf{k}^{\prime}, t=0\right)\right\rangle .
\end{aligned}
$$

Here, $\mathbf{k}$ - and $\mathbf{k}^{\prime}$-dependent functions are understood to be spatial Fourier transforms with respect to $\mathbf{r}$ and $\mathbf{r}^{\prime}$, respectively. Now suppose that there is at most a single fluorescent colloidal particle inside the confocal volume. The Fourier transform of the dye concentration $c_{d}$ can then be written as,

$$
\begin{aligned}
c_{d}(\mathbf{k}, t)= & B_{f}(\mathbf{k}) \exp \left\{i \mathbf{k} \cdot \mathbf{r}_{c}(t)\right\}, \\
& \text { where } B_{f}(\mathbf{k})=\int_{V_{0}(t)} d \mathbf{r} \chi_{d}(\mathbf{r}) \exp \{i \mathbf{k} \cdot \mathbf{r}\} .
\end{aligned}
$$

Here, $\mathbf{r}_{c}$ is the position coordinate (the position of the centerof-mass) of the colloidal particle, $B_{f}$ is referred to as the "fluorescence amplitude," with $V_{0}$ the volume occupied by the colloidal sphere with its center of mass at the origin (as indicated by the subscript " 0 "), and $\chi_{d}(\mathbf{r})$ is the characteristic function for the dye on the surface and within the core of the colloidal particle $\left[\chi_{d}(\mathbf{r})=1\right.$ when there is a dye molecule at $\mathbf{r}$, and $=0$ otherwise $]$. Note that the fluorescence amplitude in Eq. (30) is time independent when the distribution of fluorescent dye is spherically symmetric. For translationally invariant systems (with $\delta$ the delta distribution) and in the long-time limit, one has,

$$
\begin{aligned}
\left\langle c_{d}(\mathbf{k}, t) c_{d}\left(\mathbf{k}^{\prime}, t=0\right)\right\rangle & \\
& \sim \delta\left(\mathbf{k}+\mathbf{k}^{\prime}\right)[B(\mathbf{k})]^{2}\left\langle\exp \left\{i \mathbf{k} \cdot\left[\mathbf{r}_{c}(t)-\mathbf{r}_{c}(t=0)\right]\right\}\right\rangle \\
\quad & \delta\left(\mathbf{k}+\mathbf{k}^{\prime}\right)[B(\mathbf{k})]^{2} \exp \left\{-D_{s} k^{2} t\right\},
\end{aligned}
$$

where $D_{s}$ is the long-time self-diffusion coefficient. This expression is valid when the tracer particle resides in the confocal volume for sufficiently long times, such that long-time diffusive behavior is attained. It thus follows from Eq. (29) that,

$$
C_{f}(t) \sim \int d \mathbf{k} I_{0}^{2}(\mathbf{k})\left|B_{f}(\mathbf{k})\right|^{2} \exp \left\{-D_{s} k^{2} t\right\} .
$$

Note that the ensemble average in Eq. (32) for the fluorescence autocorrelation function is a $k$-weighted average of the electric field autocorrelation function that is measured in a dynamic light scattering experiment.

Assuming small spheres, such that $|B(k)|^{2} \approx 1$ for the wave vectors of interest in the integral in Eq. (32), and from the Fourier transform of the confocal intensity in Eq. (27), it follows by integration that,

$$
C_{f}(t) \sim\left[1+\frac{D_{s} t}{\sigma_{1}^{2}}\right]^{-1}\left[1+\frac{D_{s} t}{\sigma_{2}^{2}}\right]^{-1 / 2} .
$$

The fluorescent intensity autocorrelation function thus decays algebraically with time, with two characteristic time constants $\sigma_{1,2}^{2} / D_{s}$. The geometrical constants $\sigma_{1,2}$ can be determined from a measurement of a dilute system where $D_{s}$ is equal to the Einstein diffusion constant, that can be obtained independently from dynamic light scattering.

When the particles are not small, the fluorescent intensity is nonzero already when the outer part of a particle enters the confocal volume. At that moment the center of the colloid is still outside the confocal volume. In such cases the $k$ dependence of the fluorescence amplitudes in Eq. (32) comes into play. For homogeneously labeled spheres, $|B(k)|$ can be represented quite accurately by a Gaussian $\sim \exp \left\{-0.11 k^{2} a^{2}\right\}$, up to wave vectors where $B(k)$ is small enough that it does not contribute anymore to the integral over k. Here, $a$ is the radius of the spherical colloidal particle. We thus obtain the "effective size" of the confocal volume as

$$
\sigma_{1,2}^{\text {eff }}=\sqrt{\sigma_{1,2}^{2}+0.22 a^{2}} .
$$

This effective increase of the confocal volume quantifies the finite fluorescent intensity before the center of the sphere actually entered the confocal volume.

Experimental correlation functions show some features that we have not discussed above. First of all, in the various steps taken in the above analysis, prefactors are always omitted. It turns out, by including all prefactors, that the proportionality constant in Eq. (33) is equal to 1/[the average number of fluorescent colloidal particles in the confocal volume]. This reflects the relative decrease of number fluctuations in a given volume with increase of volume. Second, there may be free dye molecules, not attached to colloidal particles, in the solvent. When this is the case, there is a second, additive contribution of the form (33), except that the time constants are much smaller than those for the colloidal particles. Free dye is therefore seen only at very small times. Third, dye molecules that are excited in long lived triplet states, do not contribute to the fluorescent intensity and therefore temporarily reduce the number of fluorescent dye molecules. Triplet state excitation can thus be regarded as "reversible bleaching." The relative amplitude of such contributions depends on the sort of dye that is used, as well as the concentration of dye on the surface and/or inside the core of a colloidal particle. For our samples, triplet state contributions were found to be very small. In addition, these very small triplet contributions decay on a time scale that is orders of magnitude faster than the diffusion times of the colloidal spheres.

The above analysis is a simplified version of a more elaborate analysis in Ref. 44. Overviews of FCS and its application to biological systems can be found in Refs. 45-49. An extensive literature list can be found on the FCS website of Zeiss.

\section{B. Dynamic light scattering}

In order to verify whether the true long-time limit of the tracer diffusion coefficient is measured in the FCS experi- 
ments, we performed dynamic light scattering experiments. For tracer systems, dynamic light scattering can be used to measure the entire time dependence of the mean-squared displacement, allowing for an unambiguous determination of the long-time limit of the self-diffusion coefficient. The necessary condition to probe self-diffusion of the spheres is that the scattered intensity of the rods is very much smaller than that of the spheres, and that the concentration of the spheres is so low that a sphere does not interact with other spheres. This limits the applicability of dynamic light scattering to intermediate sized spheres, for radii $a$ in the range $50 \mathrm{~nm}$ $<a<500 \mathrm{~nm}$. For smaller spheres the scattered intensity of the rods can no longer be neglected. For larger spheres the scattered intensity for typical experimentally accessible scattering angles is also too small as compared to the scattered intensity by the rods, while extrapolations must be done from relatively small scattering angles.

The procedure to obtain the time-dependent meansquared displacement from dynamic light scattering data is as follows (see, for example, Refs. 50 and 51). When the intensity of the tracer spheres dominates and the spheres do not mutually interact, the measured normalized electric field autocorrelation function $g_{1}(k, t)$ is equal to,

$$
g_{1}(k, t)=\langle\exp \{i \mathbf{k} \cdot[\mathbf{r}(t)-\mathbf{r}(0)]\}\rangle,
$$

where $\mathbf{k}$ is the scattered wavevector and $\mathbf{r}(t)$ is position of a tracer sphere at time $t$. For translationally and rotationally invariant systems, an expansion with respect to small wave vectors leads to,

$$
g_{1}(k, t)=\exp \left\{-\frac{1}{6} W(t) k^{2}+“ O\left(k^{4}\right),,\right\},
$$

where,

$$
W(t)=\left\langle|\mathbf{r}(t)-\mathbf{r}(0)|^{2}\right\rangle \equiv 6 D_{s}(t) t,
$$

is the mean-squared displacement. The second equation defines the time-dependent self-diffusion coefficient. The longtime self-diffusion coefficient is the long-time limit of $D_{s}(t)$, which is obtained from the slope of $W(t)$ as a function of $t$ at sufficiently large times. Hence, plotting $-6 \ln \left\{g_{1}(k, t)\right\} / k^{2}$ for a given time as a function of $k^{2}$, and extrapolating to $k=0$, yields $W(t)$ for the particular time under consideration. Typically this procedure is applied for about 50 linearly spaced times. The long-time self-diffusion coefficient $D_{s}$ then follows from $d W(t) / d t=6 D_{s}$ for times much larger than typical microstructural relaxation times of the host rods.

\section{Video microscopy}

For video microscopy we used the method described in Refs. 52 and 53. Video Microscopy is used for the $500 \mathrm{~nm}$ spheres, with a Carl Zeiss AxioCam camera and AxioVison 3.0 software to measure particle coordinates. The was Carl Zeiss plan-apochromat $20 X, N . A$. 0.75 , and $256 \times 324$ pixels black and white enhanced gain contrast TIFF image formats are stored in real time for 100 consecutive shots. The size of a pixel is $0.260 \mu \mathrm{m}$ while frames were recorded at a speed of $0.23 \mathrm{~s}$ per frame with the exposure time of the camera in the range 4-7 ms. The samples are contained in a microslide (VITRO Dynamics Inc. Cat. No. 3520), with a thickness of
TABLE I. Overview of tracer sphere sizes, applied methods, and experimental versus theoretical values for $\alpha$. An " $\mathrm{X}$ " in this table indicates that a methods has been applied for the particular tracer sphere. VM stands for video microscopy.

\begin{tabular}{ccccccr}
\hline \hline Radius (nm) & $q=L / 2 a$ & FCS & DLS & VM & $\alpha^{\text {expt }}$ & $\alpha^{\text {theor }}$ \\
\hline 500 & 0.88 & & & $X$ & $2600 \pm 500$ & 2900 \\
210 & 2.10 & $X$ & $X$ & & $2300 \pm 500$ & 830 \\
35 & 12.6 & $X$ & $X$ & & $160 \pm 40$ & 67 \\
3 & 147 & $X$ & & & $38 \pm 10$ & 11 \\
\hline \hline
\end{tabular}

$200 \mu \mathrm{m}$. The focus is in the middle of the cuvette to avoid the effects of interactions with the walls. An IDL particletracking program is used to calculate mean squared displacements as functions of time from two-dimensional (2D) images.

\section{EXPERIMENTAL RESULTS}

Four different tracer particles have been used, with radii $500,210,35$, and $3 \mathrm{~nm}$. Depending on the size of these spheres, suitable techniques to measure long-time selfdiffusion coefficients are video microscopy, DLS, and FCS. For the $500 \mathrm{~nm}$ spheres we used video microscopy to measure the long-time self-diffusion coefficient, for the 210 and $35 \mathrm{~nm}$ spheres both DLS and FCS were employed, while the $3 \mathrm{~nm}$ spheres we used FCS. The suitability of DLS depends on the ratio of the scattered light by the tracer spheres and host rods, and the accessible wave vector range. This limits the suitability of DLS to the 210 and $35 \mathrm{~nm}$ particles: the 3 $\mathrm{nm}$ particles do not scatter enough as compared to the fd rods to prepare a tracer system, while the form factor of the 500 $\mathrm{nm}$ spheres goes to zero within a too small wave vector range to be able to do the extrapolation to zero wave vector, as discussed before. For the $210 \mathrm{~nm}$ spheres extrapolations are done from the scattering angle range $15^{\circ}-60^{\circ}$, and for the $35 \mathrm{~nm}$ particles from $25^{\circ}-120^{\circ}$ in steps of $5^{\circ}$, where two independent measurements are averaged. FCS is difficult to perform for the $500 \mathrm{~nm}$, since the number density at a given low volume fraction is low and diffusion through the confocal volume takes a relatively long time.

As will become clear in the following, the combination of the three techniques is a fertile combination in order to study long-time diffusive behavior over a large range of tracer particle radii. An overview of the tracer sphere sizes and the applied techniques is given in the table.

Diffusion coefficients as measured by means of FCS cannot always be identified as long-time diffusion coefficients. This is illustrated in Fig. 4, where both the long- and short-time diffusion coefficients as obtained from DLS and diffusion coefficients obtained from FCS are plotted as functions of the fd concentration. Clearly, for small fd concentrations FCS measures short-time diffusion, whereas for high fd concentrations the FCS diffusion coefficient seems to become equal to the long-time diffusion coefficient. The reason that FCS does not measure long-time diffusion can be seen from Fig. 5(a), where the mean squared displacement of the $210 \mathrm{~nm}$ spheres as obtained from DLS experiments is plotted for various fd concentrations. As can be seen, the mean 


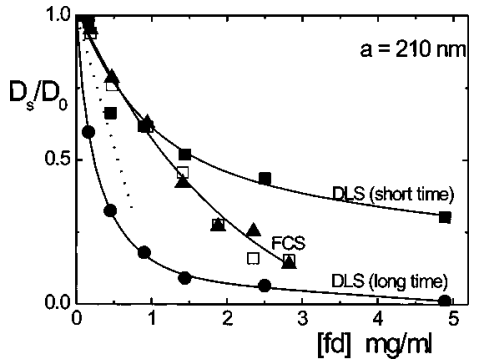

FIG. 4. Diffusion coefficients of the $210 \mathrm{~nm}$ particles as measured by DLS and FCS, as indicated in the figure. Filled symbols refer to high salt concentration ( $20 \mathrm{mM}$ TRIS $+100 \mathrm{mM} \mathrm{NaCl}$, ionic strength is $110 \mathrm{mM})$, open symbols to low salt concentration (20 mM TRIS without added salt, ionic strength is $10 \mathrm{mM}$ ). Diffusion coefficients are normalized to the Einstein diffusion coefficient $D_{0}$. The dotted line corresponds to the slope predicted by Eqs. (1) and (24).

squared displacement is $\sim 0.05 \mu \mathrm{m}^{2}$ before the long-time limit is reached for the smaller concentrations. The linear dimensions of the confocal volume [as defined by the parameters $\sigma_{1,2}$ in Eq. (27)] are $\sigma_{1} \approx 0.2 \mu \mathrm{m}$ and $\sigma_{2} \approx 0.8 \mu \mathrm{m}$. Hence, the minimum mean-squared displacement to reach the long-time diffusive limit is of the order of the squared linear dimension of the confocal volume. A $210 \mathrm{~nm}$ tracer sphere therefore diffuses through the confocal volume without reaching its long-time limit. FCS then measures the short-time diffusion coefficient (similar considerations concerning FCS measurements can be found in Ref. 16). For higher fd concentration, long-time diffusive behavior is
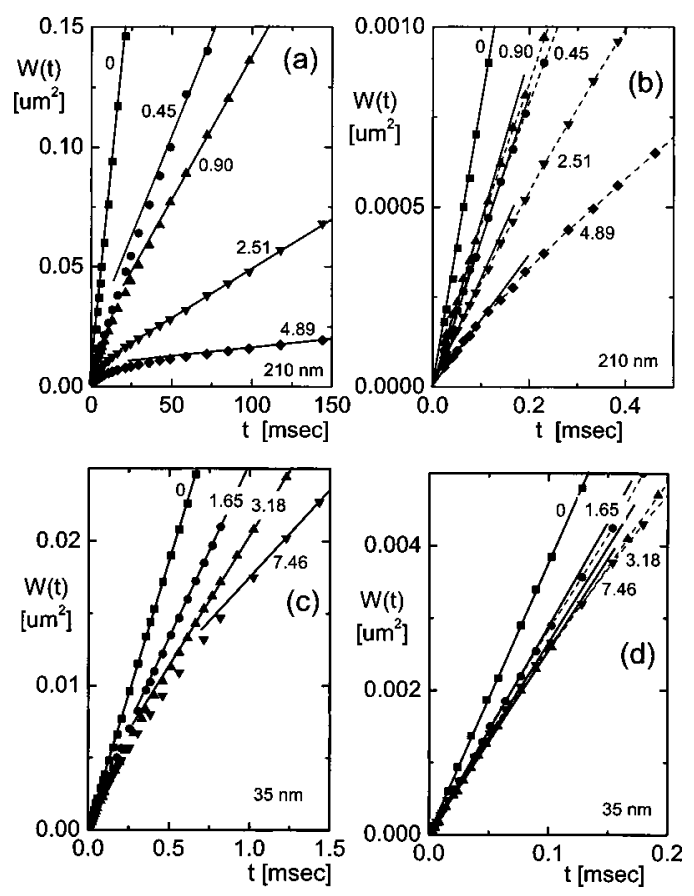

FIG. 5. The mean squared displacement $W(t)$ obtained from DLS of the 210 and $35 \mathrm{~nm}$ tracer spheres for various fd concentrations, as indicated in the figure [in units of $\mathrm{mg} / \mathrm{ml}$ ]: (a) long times, $210 \mathrm{~nm}$, (b) short times, 210 $\mathrm{nm}$, (c) long times, $35 \mathrm{~nm}$, (d) short times, $35 \mathrm{~nm}$. The solid lines correspond to slopes of $W(t)$ at long $(\mathrm{a}, \mathrm{c})$ and short $(\mathrm{b}, \mathrm{d})$ time diffusion regimes. The dashed curves in Figs. (b,d) are drawn to guide the eye. Long-time diffusion coefficients are determined from slopes up to times of about two times the time interval displayed in Figs. (a,c).
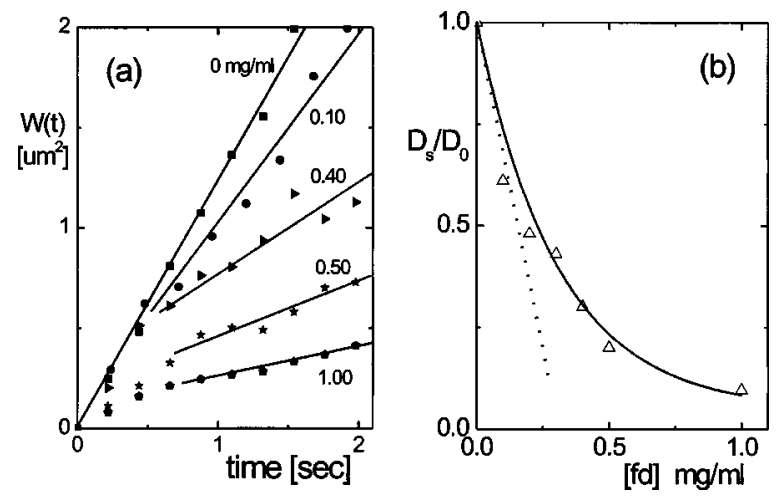

FIG. 6. (a) The mean squared displacement for the $500 \mathrm{~nm}$ tracer spheres as measured by video microscopy, at low salt concentration $(20 \mathrm{mM}$ TRIS buffer without added salt). The numbers refer to fd concentrations in $\mathrm{mg} / \mathrm{ml}$. For clarity, the time range is plotted for a limited time range. Long-time self-diffusion coefficients are obtained from a time range up to $5 \mathrm{~s}$. (b) The long-time self-diffusion coefficient as a function of fd concentration as obtained from the slopes of the solid lines that fit the long-time part of the mean squared displacements in Fig. a. The dotted line corresponds to the predicted slope from Eqs. (1) and (24) while the solid line is a guide to the eye.

reached for a mean-squared displacement of $\sim 0.01 \mu \mathrm{m}^{2}$, which is small compared to the squared linear dimension of the confocal volume, so that FCS now probes long-time diffusive motion. As can be seen from Fig. 5(c), where the mean-squared displacement is plotted for the $35 \mathrm{~nm}$ spheres, the long-time limit is reached for much smaller displacements for all concentrations, so that FCS probes long-time diffusion for these smaller spheres (FCS and DLS long-time data are indeed shown in Fig. 7 to coincide). This will also be the case then for the $3 \mathrm{~nm}$ spheres. Figures 5(b) and 5(c) show mean squared displacements for a shorter time interval, which allows for the determination of short-time diffusion coefficients.

Note that in Fig. 4 short-time self diffusion is found to be much less hindered by the rods as compared to long-time diffusion, in accordance to what has been found in Refs. 14 and 15. Short-time rotational diffusion, however, is affected by the rods in a way that is comparable to long-time translational diffusion. ${ }^{15}$

Mean-squared displacements and diffusion coefficients as a function of fd concentration of the $500 \mathrm{~nm}$ spheres as obtained from video microscopy are given in Figs. 6(a) and 6(b), respectively. Mean-squared displacements are averages over 15 tracer spheres. Note the relatively large value of the mean-squared displacement before long-time diffusive behavior is found. Here, FCS would probably probe short-time diffusion for all $\mathrm{fd}$ concentrations. The dotted line in Fig. 6(a) is the predicted slope from Eqs. (1) and (24). As can be seen, the theoretical prediction is in accordance with the experiments. For the smaller spheres, the predicted slope is about a factor of 2-3 smaller than the experimental slope: see the dotted lines in Figs. 4 and 7. The table contains an overview of the experimentally obtained values $\alpha^{\exp }$ for the slope $\alpha$ and the value $\alpha^{\text {theor }}$ as predicted by theory. The discrepancy between experiment and theory for the smaller particles is probably due to the neglect of hydrodynamic interactions in our theory. Hydrodynamic interactions of the 


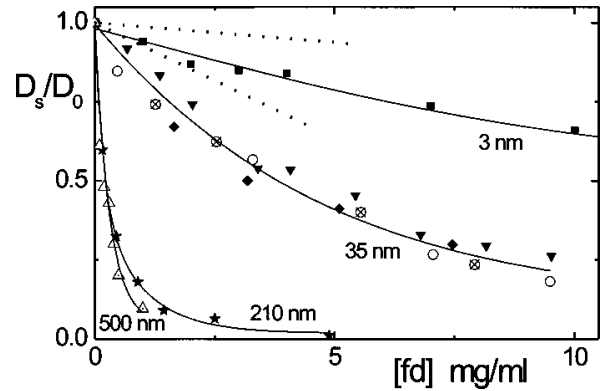

FIG. 7. The long-time self-diffusion coefficients, normalized to the Einstein diffusion coefficient $D_{0}$, as measured by FCS $(\boldsymbol{\square}, \boldsymbol{\nabla}, \otimes$, and $\bigcirc)$, DLS $(\diamond, \star)$, and video microscopy $(\triangle)$ for various tracer sphere sizes, as indicated in the figure. Solid symbols refer to high salt concentrations $(20 \mathrm{mM}$ TRIS buffer $+100 \mathrm{mM} \mathrm{NaCl}$ ), open symbols refer to low salt concentrations $(20 \mathrm{mM}$ TRIS buffer without added salt). $\otimes$ refers to a tracer volume fraction of $8.5 \times 10^{-4}$ and $\bigcirc$ to $1.3 \times 10^{-3}$. All other volume fractions of tracer spheres are less than $1.0 \times 10^{-3}$. The solid lines are guides to the eye. The dotted lines correspond to the predicted slope from Eqs. (1) and (24). The corresponding theoretical dotted lines for the two larger spheres are shown in Figs. 4 and 6.

tracer sphere with the rods become relatively more important as compared to direct interactions when the tracer sphere is smaller.

Long-time diffusion coefficients as functions of fd concentration for the various tracer spheres are collected in Fig. 7. All filled symbols relate to high salt concentration $(20 \mathrm{mM}$ TRIS + $100 \mathrm{mM} \mathrm{NaCl}$ ), while the open symbols for the 35 $\mathrm{nm}$ spheres are measured at low salt concentration $(20 \mathrm{mM}$ TRIS without adding salt). As can be seen, there is no difference between high and low salt for the $35 \mathrm{~nm}$ spheres, so that the hard core limit at these salt concentrations is reached for these spheres, and also for the larger spheres. In view of the fact that the Debye length at high salt is about $0.9 \mathrm{~nm}$, the $3 \mathrm{~nm}$ spheres are also expected to behave as hard spheres. The large spheres were found to be unstable at high salt concentration. The Debye length at low salt is so small in comparison to $500 \mathrm{~nm}$, that these spheres most probably also behave as hard spheres. The two types of open symbols for the $35 \mathrm{~nm}$ spheres relate to two concentrations of tracer spheres, complying with volume fractions of $8.5 \times 10^{-4}(\otimes)$ and $1.3 \times 10^{-3}(\bigcirc)$. As can be seen, the volume fraction of the spheres is small enough to neglect mutual interactions, so that true self diffusion is measured. The volume fraction of tracer spheres was less than $1.0 \times 10^{-3}$ for all other samples.

On various occasions we referred to diffusion of small tracer spheres as diffusion through the meshes of a network spanned by the fd rods. There are three parameters of importance for diffusion of spheres through networks: (i) the size ratio $\alpha / \xi$, where $\xi$ is the mesh size of the network, (ii) the size ratio $a / D$, and (iii) the dynamical properties of the network itself which couples to the motion of the sphere. When $a / D$ is large and the dynamics of the network is insignificant for diffusion of the tracer sphere, $D_{s} / D_{0}$ should be a function of the single parameter $a / \xi$. For cross-linked networks, it has been shown experimentally for various kinds of systems, that $D_{s} / D_{0}$ indeed scales with $a / \xi$. Except for a mode coupling approach that is valid only in the vicinity of the isotropic-nematic phase transition, ${ }^{29,30}$ the existing

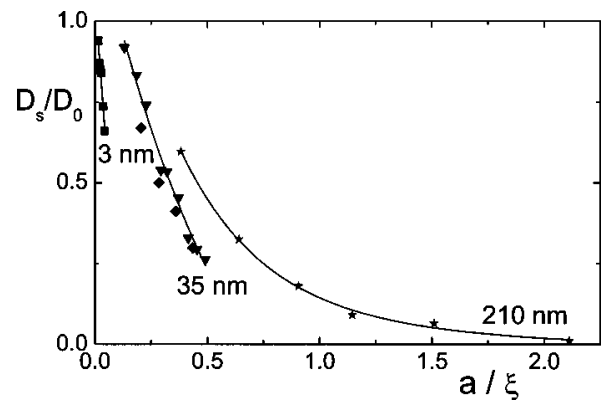

FIG. 8. The long-time self-diffusion coefficients, normalized to the Einstein diffusion coefficient $D_{0}$, as functions of $a / \xi$ for various tracer sphere sizes, as indicated in the figure.

theories $^{25-28}$ treat diffusion of spheres in rod networks with the neglect of the dynamics of the network itself. In that case this scaling is also predicted by theory. Scaling with $a / \xi$ is not found for fd-virus suspensions, as can be seen from Fig. 8. ${ }^{54}$ Even for the two tracer spheres for which $a / D$ is large, there is no scaling. This implies that the dynamics of the network is responsible for the absence of scaling. Failure of such a scaling is also found for diffusion in suspensions of "living polymers". 14 In this reference, the modified scaling relation $D_{s} / D_{0}=\left(\eta_{0} / \eta\right)\left[1+600(\xi / a)^{6}\right]$ is postulated (where $\eta_{0}$ is the shear viscosity of pure solvent, and $\eta$ is the stationary zero-shear viscosity of the suspension of living polymer). This relation fits experimental results for two different tracer sphere sizes in Ref. 14 (250 and $500 \mathrm{~nm}$ ). For very large spheres, $D_{s} / D_{0}=\eta_{0} / \eta$, as it should. However, for very small tracer spheres one should have $D_{s} / D_{0} \approx 1$. The long-time self-diffusion coefficient in Fig. 7 for the small particles is indeed seen to be very weakly depending on the fd concentration. The empirical modified scaling relation does not comply with the latter limiting behavior. A theory for diffusion of spheres through dynamical networks is still lacking. The variational approach discussed above may be a way to develop such a theory.

\section{SUMMARY AND CONCLUSION}

The systematic study of long-time self-diffusion of tracer spheres in fd-rod dispersions has been possible by combining various experimental techniques, FCS (for small tracers), DLS (for intermediate sized tracers), and video microscopy (for large tracers). For larger spheres, it is shown that FCS does not measure long-time self-diffusion. DLS experiments show that this is due to the fact that the tracer sphere diffuses through the confocal volume in a time interval that is too short to attain long-time limiting diffusive behavior.

Long-time self-diffusion of spherical tracers in rod dispersions is found to strongly depend on the size of the spheres relative to the length of the rods. This strong size dependence is not the result of stronger hinderance of motion of the tracer by a network formed by the rods, but is already evident below the overlap concentration, where the tracer sphere predominantly interacts with mutually uncorrelated 
rods. The experimentally found initial slope of $D_{s}$ versus the rod concentration increases by orders of magnitude on varying the tracer sphere radius from 3 to $500 \mathrm{~nm}$, corresponding to $q=L / 2 a=147-0.88$.

Quantitative agreement between the theoretically predicted slope of $D_{s}$ versus concentration is found for the 500 $\mathrm{nm}$ tracer spheres. For the smaller spheres, the theoretical slope is a factor of 2-3 smaller than the experimentally found slope. This is probably due to the neglect of hydrodynamic interactions between the tracer sphere and the rods, which become relatively more important as compared to direct interactions for such smaller spheres.

For concentrations of rods much higher than the overlap concentration, diffusion can be discussed in terms of diffusion through a network which is characterized by a certain mesh size $\xi$. The few theories that exist, however, neglect the dynamics of the network and assume the existence of permanent cross links between the rods or describe the network as a porous medium. In that case $D_{s}$ is predicted to be a function of the size ratio $a / \xi$ (with $a$ the radius of the tracer sphere and $\xi$ the mesh size). This kind of scaling is indeed found in experiments on cross-linked networks. These theories, however, do not incorporate spatial inhomogeneities of the cross-linked network, which gives rise to the experimentally found anomalous diffusive behavior. The network spanned by the fd rods is a dynamical network, where the rods are not cross linked to each other. A strong deviation from scaling with $a / \xi$ is found in the present experiments. It follows that the dynamics of the fd-rod network itself is very important. So far, there is no theory that takes such network dynamics into account. The variational approach developed here might be extended to diffusion through a dynamical network of rods.

\section{ACKNOWLEDGMENTS}

The authors thank N. A. M. Verhaegh, C. M. van Kats, and G. Bosma from the Utrecht University for providing rhodamine-labeled silica spheres. Financial support from the DAAD and KBN is gratefully acknowledged. Part of this work was done within the framework of the SoftComp Network of Excellence.

\section{APPENDIX A: DERIVATION OF THE EXPLICIT FORM OF THE FUNCTIONAL}

Some of the mathematical steps involved to arrive at Eq. (18) for the functional in Eq. (17) will be outlined in this appendix. First introduce the separation $\mathbf{R}=\mathbf{R}_{1}-\mathbf{R}_{2}$ between the centers of the sphere and a rod in units of the thickness $D$ of the rod. Substitution of the definitions of the operators yields ( $V$ is the volume of the system and $\boldsymbol{\nabla}$ is the gradient operator with respect to $\mathbf{R}$ ),

$$
\begin{aligned}
\left\langle\Psi \mid \hat{\mathcal{L}}_{B}^{(0)} \Psi\right\rangle= & V D^{3} \int d \mathbf{R} \oint d \hat{\mathbf{u}} P^{\mathrm{eq}} \Psi \\
& \times\left\{\left(1+\epsilon-\frac{1}{3} \epsilon_{t}\right)(\boldsymbol{\nabla}-\beta[\boldsymbol{\nabla} V]) \cdot \boldsymbol{\nabla} \Psi\right. \\
& +\epsilon_{t}(\boldsymbol{\nabla}-\beta[\boldsymbol{\nabla} V]) \cdot \hat{\mathbf{u}} \hat{\mathbf{u}} \cdot \boldsymbol{\nabla} \Psi \\
& \left.+\epsilon_{r}(\hat{\mathcal{R}}-\beta[\hat{\mathcal{R}} V]) \cdot \hat{\mathcal{R}} \Psi\right\} .
\end{aligned}
$$

Using Gauss's integral theorem and using that $P^{\mathrm{eq}}$ $\sim \exp \{-\beta V\}$, it is easily verified that,

$$
\begin{aligned}
\int d \mathbf{R} P^{\mathrm{eq}} \Psi \boldsymbol{\nabla} \cdot \boldsymbol{\nabla} \Psi= & -\int d \mathbf{R} P^{\mathrm{eq}}\left\{|\boldsymbol{\nabla} \Psi|^{2}\right. \\
& +\beta \Psi[\boldsymbol{\nabla} \Psi] \cdot[\boldsymbol{\nabla} V]\},
\end{aligned}
$$

so that the integral corresponding to the first term within the curly brackets in the integrand in Eq. (A1) is found to be equal to,

$$
\int d \mathbf{R} P^{\mathrm{eq}} \Psi(\boldsymbol{\nabla}-\beta[\boldsymbol{\nabla} V]) \cdot \boldsymbol{\nabla} \Psi=-\int d \mathbf{R} P^{\mathrm{eq}}|\boldsymbol{\nabla} \Psi|^{2} .
$$

The integral corresponding to the second term is reduced similarly as,

$$
\begin{gathered}
\int d \mathbf{R} P^{\mathrm{eq}} \Psi(\nabla-\beta[\nabla V]) \cdot \hat{\mathbf{u}} \hat{\mathbf{u}} \cdot \nabla \Psi \\
=-\int d \mathbf{R} P^{\mathrm{eq}}|\hat{\mathbf{u}} \cdot \boldsymbol{\nabla} \Psi|^{2} .
\end{gathered}
$$

The last integral can be treated similarly, using that,

$$
\oint d \hat{\mathbf{u}} \hat{\mathcal{R}}(\cdots)=0
$$

which is a direct consequence of Stokes's theorem. It follows from this theorem, similar to Eq. (A2), that,

$$
\begin{aligned}
& \oint d \hat{\mathbf{u}} P^{\mathrm{eq}} \Psi \hat{\mathcal{R}} \cdot \hat{\mathcal{R}} \Psi \\
& =-\oint d \hat{\mathbf{u}} P^{\mathrm{eq}}\left[|\hat{\mathcal{R}} \Psi|^{2}+\beta \Psi[\hat{\mathcal{R}} \Psi] \cdot[\hat{\mathcal{R}} V]\right],
\end{aligned}
$$

and hence, similar to Eq. (A3),

$$
\oint d \hat{\mathbf{u}} P^{\mathrm{eq}} \Psi(\hat{\mathcal{R}}-\beta[\hat{\mathcal{R}} V]) \cdot \hat{\mathcal{R}} \Psi=-\oint d \hat{\mathbf{u}} P^{\mathrm{eq}}|\hat{\mathcal{R}} \Psi|^{2} .
$$

The bilinear contribution (A1) to the functional can thus be written as,

$$
\begin{aligned}
\left\langle\Psi \mid \hat{\mathcal{L}}_{B}^{(0)} \Psi\right\rangle= & -V D^{3} \int d \mathbf{R} \oint d \hat{\mathbf{u}} P^{\mathrm{eq}} \\
& \times\left\{\left(1+\epsilon-\frac{1}{3} \epsilon_{t}\right)|\boldsymbol{\nabla} \Psi|^{2}+\epsilon_{t}|\hat{\mathbf{u}} \cdot \boldsymbol{\nabla} \Psi|^{2}\right. \\
& \left.+\epsilon_{r}|\hat{\mathcal{R}} \Psi|^{2}\right\} .
\end{aligned}
$$

Next, consider the linear contribution to the functional (17), 


$$
\begin{aligned}
& 2\left\langle\Psi \mid\left[P^{\mathrm{eq}}\right]^{-1} \hat{\mathcal{L}}^{(1)} P^{\mathrm{eq}}\right\rangle \\
& =-2 \epsilon V D^{3} \hat{\mathbf{e}}_{3} \cdot \int d \mathbf{R} \oint d \hat{\mathbf{u}} \Psi \nabla P^{\mathrm{eq}},
\end{aligned}
$$

where $\hat{\mathbf{e}}_{3}=(0,0,1)$. Since $P^{\mathrm{eq}}$ is a constant, independent of $\mathbf{R}$ for $R \geqslant(L+2 a) / 2 D$, the integral with respect to $\mathbf{R}$ does not extend beyond $R=(L+2 a) / 2 D$. Hence, applying Gauss's integral theorem,

$$
\begin{aligned}
\int d \mathbf{R} \Psi \nabla P^{\mathrm{eq}}= & -\int_{R<(L+2 a) / 2 D} d \mathbf{R} P^{\mathrm{eq}} \nabla \Psi \\
& +\left(\frac{L+2 a}{2 D}\right)^{2} \oint_{R=(L+2 a) / 2 D} d \hat{\mathbf{R}} \hat{\mathbf{R}} \Psi P^{\mathrm{eq}}
\end{aligned}
$$

where $\oint d \hat{\mathbf{R}}(\cdots)$ denotes integration over the angular coordinates of $\mathbf{R}$ over a spherical surface with radius $R=(L$ $+2 a) / 2 D$, and $\hat{\mathbf{R}}=\mathbf{R} / R$. Substitution into Eq. (A9), thus leads to,

$$
\begin{aligned}
& 2\left\langle\Psi \mid\left[P^{\mathrm{eq}}\right]^{-1} \hat{\mathcal{L}}^{(1)} P^{\mathrm{eq}}\right\rangle \\
&=2 \epsilon V D^{3} \oint d \hat{\mathbf{u}} P^{\mathrm{eq}}\left[\int_{R<(L+2 a) / 2 D} d \mathbf{R} \frac{\partial}{\partial Z} \Psi\right. \\
&\left.-\left(\frac{L+2 a}{2 D}\right)^{2} \oint_{R=(L+2 a) / 2 D} d \hat{\mathbf{R}} \frac{Z}{R} \Psi\right]
\end{aligned}
$$

Apart from the irrelevant prefactor $V D^{3} P^{\mathrm{eq}} / \chi=D^{3} / 4 \pi V$, this reproduces the expression in Eq. (18) [note that $\chi=1$ for $R=(L+2 a) / 2 D]$.

\section{APPENDIX B: DERIVATION OF EQ. (23)}

The ensemble average of the pair-interaction potential in Eq. (6) for the stationary velocity of the tracer sphere is equal to,

$$
N\left\langle\nabla_{1} V\right\rangle=N V D^{2} \int d \mathbf{R} \oint d \hat{\mathbf{u}} P \nabla_{1} V,
$$

where, as before, distances are expressed in units of the thickness $D$ of the rods. Substitution of Eq. (12), using that for hard-core interactions $\nabla_{1} \chi=-\beta \chi \nabla_{1} V$ and that the volume fraction $\varphi$ of end-capped rods is equal to,

$$
\varphi=\frac{\pi}{4} D^{2} L\left(1-\frac{D}{3 L}\right)
$$

thus leads to,

$$
N\left\langle\nabla_{1} V\right\rangle=-\frac{\varphi}{\pi^{2} p\left(1-\frac{1}{3 p}\right)} F^{\mathrm{ext}} \int d \mathbf{R} \oint d \hat{\mathbf{u}} \Psi \nabla_{1} \chi .
$$

Next consider the ensemble averaged Brownian force in Eq. (6). From Eq. (12) it is immediately found that,
$N k_{B} T\left\langle\nabla_{1} \ln \{P\}\right\rangle$

$$
=\frac{\varphi}{\pi^{2} p\left(1-\frac{1}{3 p}\right)} F^{\text {ext }} \int d \mathbf{R} \oint d \hat{\mathbf{u}}\left\{\Psi \nabla_{1} \chi+\chi \nabla_{1} \Psi\right\} .
$$

The first term in the curly brackets cancels against the average potential energy in Eq. (B3). Hence, the magnitude of the ensemble averaged velocity is equal to,

$\langle v\rangle=\beta D_{0} F^{\mathrm{ext}}\left[1-\frac{\varphi}{\pi^{2} p\left(1-\frac{1}{3 p}\right)} \int d \mathbf{R} \oint d \hat{\mathbf{u}} \chi \frac{\partial}{\partial Z} \Psi\right]$.

where it is used that the ensemble averaged velocity is along the $z$ direction. The effective friction coefficient $\gamma$ $=F^{\text {ext }} /\langle v\rangle$ follows immediately from this expression, from which the long-time self-diffusion $D_{s}=k_{B} T / \gamma$ follows as,

$$
D_{s}=D_{0}\left[1-\frac{\varphi}{\pi^{2} p\left(1-\frac{1}{3 p}\right)} \int d \mathbf{R} \oint d \hat{\mathbf{u}} \chi \frac{\partial}{\partial Z} \Psi\right]
$$

Using Gauss's integral theorem in order to transform the volume integral to integrals with a bounded integration range [like in Eq. (A10) in Appendix A, which is handy for numerical integration] finally leads to Eq. (23).

${ }^{1}$ S. Gorti and B. R. Ware, J. Chem. Phys. 83, 6449 (1985).

${ }^{2}$ J. D. Jones and K. Luby-Phelps, Biophys. J. 71, 2742 (1996).

${ }^{3}$ C. F. Schmidt, M. Bärmann, G. Isenberg, and E. Sackmann, Macromolecules 22, 3638 (1989).

${ }^{4}$ O. Seksek, J. Biwersi, and A. S. Verkman, J. Cell Biol. 138, 131 (1997).

${ }^{5}$ Y. Shafrir, D. ben-Avraham, and G. Forgacs, J. Cell. Sci. 113, 2747 (2000)

${ }^{6}$ M. Arrio-Dupont, G. Foucault, M. Vacher, P. F. Deveaux, and S. Cribier, Biophys. J. 78, 901 (2000).

${ }^{7}$ J. Apgar, Y. Tseng, E. Fedorov, M. B. Herwig, S. C. Almo, and D. Wirtz, Biophys. J. 79, 1095 (2000).

${ }^{8}$ Y. Tseng and D. Wirtz, Biophys. J. 81, 1643 (2001).

${ }^{9}$ A. S. Verkman, Trends Biochem. Sci. 27, 27 (2002).

${ }^{10}$ I. Y. Wong, M. L. Gardel, D. R. Reichman, E. R. Weeks, M. T. Valentine, A. R. Bausch, and D. A. Weitz, Phys. Rev. Lett. 92, 178101 (2004).

${ }^{11}$ D. A. Schafer and J. A. Cooper, Control of Actin Assembly at Filament Ends, http://www.cooperlab.wustl.edu/Reviews/ReviewARCB $\mathrm{M}_{\mathrm{M}} \mathrm{s} /$ ReviewARCB $\mathrm{M}_{\mathrm{S}} \mathrm{s}$ html

${ }^{12}$ H. Isambert and A. C. Maggs, Macromolecules 29, 1036 (1996).

${ }^{13}$ S. Mangenot, S. Keller, and J. Rädler, Biophys. J. 85, 1817 (2003).

${ }^{14}$ J. van der Gucht, N. A. M. Besseling, W. Knoben, L. Bouteiller, and M. A. Cohen, Phys. Rev. E 67, 051106 (2003).

${ }^{15}$ G. H. Koenderink, S. Sacanna, D. G. A. Aarts, and A. P. Philipse, Phys. Rev. E 69, 021804 (2004).

${ }^{16}$ C. Lellig, J. Wagner, R. Hempelmann, S. Keller, D. Lumma, and W. Härtl, J. Chem. Phys. 121, 7022 (2004).

${ }^{17}$ C. Graf, H. Kramer, M. Deggelmann, M. Hagenbüchle, C. Johner, C. Martin, and R. Weber, J. Chem. Phys. 98, 4920 (1993).

${ }^{18}$ F. G. Schmidt, B. Hinner, E. Sackmann, and J. X. Tang, Phys. Rev. E 62, 5509 (2000).

${ }^{19}$ T. A. J. Lenstra, Z. Dogic, and J. K. G. Dhont, J. Chem. Phys. 114, 10151 (2001). 
${ }^{20}$ K.-H. Lin, J. C. Crocker, A. C. Zeri, and A. G. Yodh, Phys. Rev. Lett. 87, 088301 (2001).

${ }^{21}$ J. Tang and S. Fraden, Liq. Cryst. 19, 459 (1995).

${ }^{22}$ M. Adams, Z. Dogic, S. L. Keller, and S. Fraden, Nature (London) 393, 349 (1998).

${ }^{23}$ Z. Dogic and S. Fraden, Langmuir 16, 7820 (2000).

${ }^{24}$ Z. Dogic, K. R. Purdy, E. Grelet, M. Adams, and S. Fraden, Phys. Rev. E 69, 051702 (2004).

${ }^{25}$ R. I. Cukier, Macromolecules 17, 252 (1984).

${ }^{26}$ A. R. Altenberger, M. Tirrell, and J. S. Dahler, J. Chem. Phys. 84, 5122 (1986).

${ }^{27}$ A. G. Ogston, B. N. Preston, and J. D. Wells, Proc. R. Soc. London 333, 297 (1973).

${ }^{28}$ J. Han and J. Herzfeld, Biophys. J. 65, 1155 (1993).

${ }^{29}$ B. Bagchi and S. Bhattacharyya, Adv. Chem. Phys. 116, 67 (2001).

${ }^{30}$ R. Vasanthi, S. Ravichandran, and B. Bagchi, J. Chem. Phys. 115, 10022 (2001).

${ }^{31}$ J. K. G. Dhont, M. P. B. van Bruggen, and W. J. Briels, Macromolecules 32, 3809 (1999).

${ }^{32}$ J. G. de la Torre and V. A. Bloomfield, Q. Rev. Biophys. 14, 81 (1981).

${ }^{33}$ I. Stakgold, Green's Functions and Boundary Value Problems (Wiley, New York, 1979).

${ }^{34}$ G. K. Batchelor, J. Fluid Mech. 52, 245 (1972); 74, 1 (1976); 131, 155 (1983); A corrigendum to the last paper can be found in 137, 467 (1983).

${ }^{35}$ B. Cichocki and B. U. Felderhof, J. Chem. Phys. 89, 1049 (1988); 94, 556 (1991).

${ }^{36}$ K. Osseo-Asare and F. J. Arriagada, Colloids Surf. 50, 321 (1990); F. J. Arriagada and K. Osseo-Asare, J. Colloid Interface Sci. 211, 210 (1999).

${ }^{37}$ N. A. M. Verhaegh, Ph.D. thesis, Utrecht University, 1996.

${ }^{38}$ N. A. M. Verhaegh and A. van Blaaderen, Langmuir 10, 1427 (1994).

${ }^{39}$ W. Stöber, A. Fink, and E. Bohn, J. Colloid Interface Sci. 26, 62 (1968).

${ }^{40}$ K. Zimmermann, J. Hagedorn, C. C. Heuck, M. Hinrichsen, and J. Ludwig, J. Biochem. (Tokyo) 261, 1653 (1986).
${ }^{41}$ J. Sambrook, E. F. Fritsch, and T. Maniatis, Molecular Cloning: A Laboratory Manual (Cold Spring Harbor Laboratory, New York, 1989).

${ }^{42}$ L. Onsager, Ann. N.Y. Acad. Sci. 51, 62 (1949).

${ }^{43}$ Z. Dogic, Ph.D. thesis, Brandeis University, 2000.

${ }^{44}$ S. R. Aragón and R. Pecora, J. Chem. Phys. 64, 1791 (1976); Biopolymers 14, 119 (1975).

${ }^{45}$ E. L. Elson, Traffic Eng. 2, 789 (2001).

${ }^{46}$ S. T. Hess, S. Huang, A. A. Heikal, and W. W. Webb, Biochemistry 41, 697 (2002).

${ }^{47}$ S. T. Hess and W. W. Webb, Biophys. J. 83, 2300 (2002).

${ }^{48}$ D. Lumma, S. Keller, Th. Vilgis, and J. Rädler, Phys. Rev. Lett. 90, 218301 (2003).

${ }^{49}$ R. Rigler and E. S. Elson, Fluorescence Correlation Spectroscopy (Springer, Berlin, 2001).

${ }^{50}$ R. Pecora, Dynamic Light Scattering; Applications of Photon Correlation Spectroscopy (Plenum, New York, 1985).

${ }^{51}$ J. K. G. Dhont, An Introduction to Dynamics of Colloids (Elsevier, Amsterdam, 1996).

${ }^{52}$ J. C. Crocker and E. Weeks, http://www.physics.emory.edu/weeks/idl/ tracking.html, "Particle tracking using IDL."

${ }^{53}$ J. C. Crocker and D. G. Grier, J. Colloid Interface Sci. 179, 298 (1996).

${ }^{54}$ Here, the mesh size is calculated as follows. Since the mesh size scales with $1 / \sqrt{c}$ [see P.-G. de Gennes, P. Pincus, and R. M. Velasco, J. Phys (Les Ulis) 37, 1461 (1976)], one can write $\xi=K / \sqrt{c}$. Just above the overlap concentration, $\xi \approx L$, so that $K \approx L \sqrt{c^{*}}$. Using the size and molar weight of fd virus, it follows that $a / \xi \approx 2 / q \sqrt{c[m g / m l]}$. Alternatively, if the unit cell of a network is taken cubic, one finds the same expression with a prefactor of $2.4 / q$ instead of $2 / q$ (see C. F. Schmidt, Dissertation, Technical University of München, 1988). Since we are interested here in scaling behavior, these minor differences in the prefactor are irrelevant. 